\title{
RADIOTHERAPY IN THE TREATMENT OF ORBITAL TUMOURS *
}

BY

\section{LEDERMAN}

Radiotherapy Department, Royal Marsden Hospital

The clinical material for this study has come to the Royal Marsden $\stackrel{\vec{\varnothing}}{\circ}$

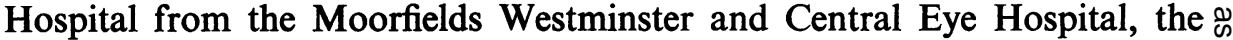
Royal Eye Hospital, the Royal National Throat, Nose and Ear Hospital, $\vec{o}$ and the Metropolitan Ear, Nose and Throat Hospital.

It is selected according to the therapy required, and cannot therefore be fairly used for assessing the incidence of orbital neoplasms. Table I shows $\frac{0}{\circ}$. the marked preponderance of secondary malignant tumours, which demon- $\overrightarrow{0}$ strates this process of selection.

\section{BENIGN TUMOURS}

These are rare (Table II) and the indications for radiotherapy are restricted 9 to haemangiomata (Figs 1 and 2, opposite). There were six orbital angiomata $\vec{P}$ without involvement of the lids, but in a series of 48 angiomata of the lids, two had some degree of intra-orbital extension. Haemangiomata in children mostly undergo spontaneous regression and the main indications for treatment are:

(1) haemorrhage or infection;

(2) situation of the lesion on the scalp, napkin area, or neighbourhood of the eye्t

(3) satisfaction of the parents, who find it hard to accept a purely expectant policy when a simple, safe, and helpful remedy is available.

TABLE I

ORBITAL TUMOURS SEEN AT

THE ROYAL MARSDEN

HOSPITAL. 1933-1955

\begin{tabular}{|c|c|c|c|}
\hline \multicolumn{3}{|c|}{ Type of Tumour } & $\begin{array}{l}\text { No. of } \\
\text { Cases }\end{array}$ \\
\hline Benign $\quad \ldots$ & $\ldots$ & $\ldots$ & 17 \\
\hline Granuloma & $\cdots$ & $\cdots$ & 5 \\
\hline \multirow{2}{*}{ Malignant } & Primary & $\ldots$ & 42 \\
\hline & Secondary & $\ldots$ & 126 \\
\hline Total & ... & $\ldots$ & 190 \\
\hline
\end{tabular}

TABLE II BENIGN TUMOURS AND GRANULOMATA

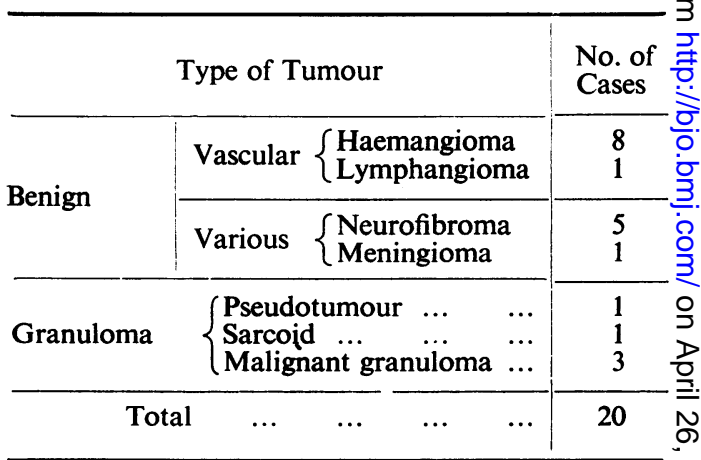

Radiotherapy is the method of choice when treatment is indicated, and it is safe because only infrequent small doses are necessary; but, whereas results $\omega_{\sigma}^{\omega}$ in lid and cutaneous haemangiomata are almost uniformly satisfactory, 


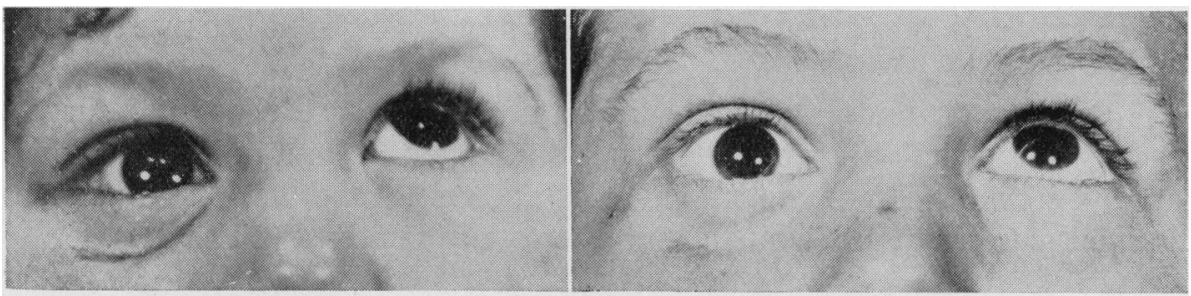

(A) Before radiation treatment.

(B) 7 years after treatment.

Operation had been attempted twice before. Movements of globe on right side restricted.

Fig. 1.-Orbital haemangioma.

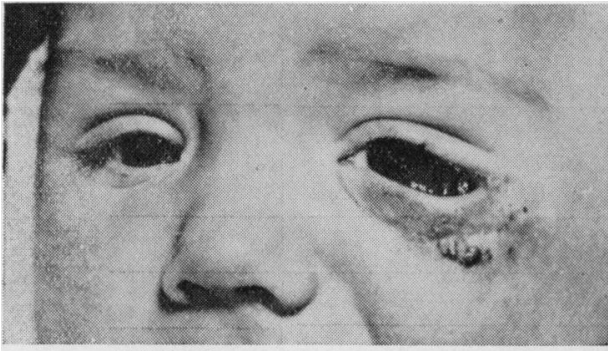

(A) Before radiation treatment.

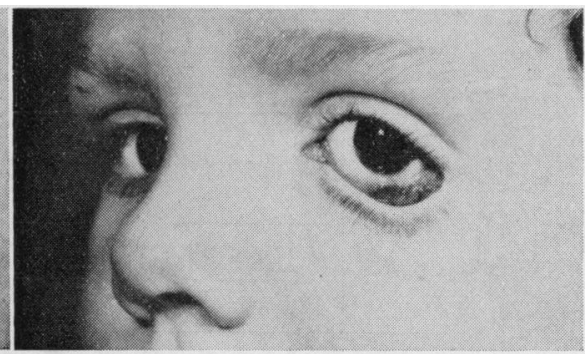

(B) 2 years after treatment.

FIG. 2.-Haemangioma of lid with involvement of orbit, recurrent after surgery.

complete resolution is not invariable in orbital cases, and surgical removal after radiotherapy was necessary in one of our patients.

\section{Malignant Granuloma}

These are necrotizing granulomatous processes of unknown aetiology usually affecting the nasal passages (Fig. 3). The outcome is usually fatal and the only remedy of occasional but proved value is radiotherapy (Ellis, 1955). These patients usually reach the rhinologist first, but of the six cases seen, three had proptosis in addition to invasion of the nasal fossa and paranasal sinuses, and in two the eye was lost.

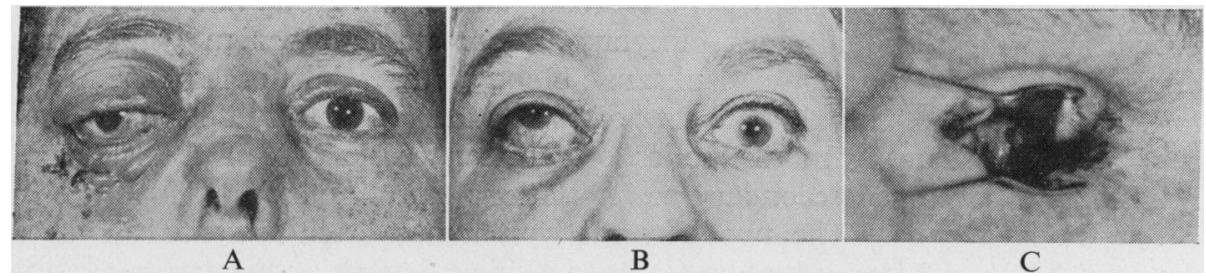

Fig. 3.-Malignant granuloma. This is primarily a disease of the nasal passages, but the orbit was invaded in three out of six patients seen. In $C$ the eye has been removed.

\section{Primary Malignant Tumours}

The diagnosis of a primary malignant orbital tumour must be confirmed histologically. All the cases in this series with one exception were so. 
confirmed, and no patient's local condition was worsened because a biopsy was performed.

The primary tumours seen by the radiotherapist are those the surgeon regards as unsuitable for operation, i.e. sarcomata of lymphoid tissue, other $\overline{\mathrm{g}}$ rapidly growing sarcomata of children and young adults, and malignant $\mathrm{O}$ lacrimal gland tumours which cannot be totally removed (Table III).

TABLE III

PRIMARY MALIGNANT TUMOURS

\begin{tabular}{|c|c|c|c|c|c|c|c|c|}
\hline \multicolumn{8}{|c|}{ Type of Tumour } & \multirow{2}{*}{$\begin{array}{c}\text { No. of Cases } \\
14\end{array}$} \\
\hline \multirow{3}{*}{ Sarcoma } & \multicolumn{3}{|c|}{ Of Lymphoid Tissue } & \multicolumn{4}{|c|}{ 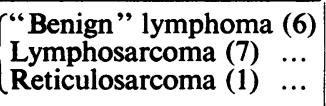 } & \\
\hline & Rhabdom & sarcor & & $\ldots$ & $\ldots$ & $\ldots$ & $\ldots$ & 7 \\
\hline & Other ... & $\ldots$ & $\ldots$ & $\ldots$ & $\ldots$ & $\ldots$ & $\ldots$ & 6 \\
\hline \multicolumn{2}{|c|}{ Lacrimal Gland $\ldots$} & $\ldots$ & $\ldots$ & $\ldots$ & $\ldots$ & $\ldots$ & $\ldots$ & 14 \\
\hline \multicolumn{4}{|c|}{ Nerve Tissue (Glioma of optic nerve) } & $\ldots$ & $\ldots$ & $\ldots$ & $\ldots$ & 1 \\
\hline & Total & ... & $\ldots$ & $\ldots$ & $\ldots$ & $\ldots$ & $\ldots$ & 42 \\
\hline
\end{tabular}

Sarcomata of Lymphoid Tissue.-These are the commonest primary orbital tumours; they are all uniformly radio-sensitive and radiotherapy is the treatment of choice. The different histological labels given to these tumours should not be allowed to obscure their histogenic unity. Their structure and behaviour vary widely, for the histologically benign lymphoma may cause rapid death from dissemination, whereas the patient with a lymphosarcoma may survive for many years. The reticulosarcoma is the least malignant of the group and has the best prognosis, whereas some cases of lymphosarcomata and benign lymphomata disseminate widely or develop a leukaemic blood picture whilst others remain well for varying periods, the ultimate fate of the patient apparently depending on a capricious providence. In this series of fourteen cases, two of the "benign" lymphomata developed secondary manifestations (chest, spine) and one patient developed leukaemia. The two bilateral lymphomata each presented laryngeal deposits (bilateral false cord infiltration and a single deposit on the aryo-eipiglottic fold). It would thus appear wise to examine the larynx routinely in all cases of ${ }^{\circ}$ orbital lymphomata.

The results (Table IV, opposite) show that the prognosis for this group of tumours $\underset{\mathfrak{N}}{\stackrel{\mathcal{W}}{ }}$ is reasonably good, and the eye should prove no problem as the only risk of damage $\stackrel{2}{2}$ is late cataract. In this series no eye was lost through radiation treatment (Figs 4,5 , and 6, opposite). 
TABLE IV

SARCOMA OF LYMPHOID TISSUE

\begin{tabular}{|c|c|c|c|c|c|c|c|c|c|c|}
\hline \multirow{2}{*}{\multicolumn{2}{|c|}{ Type of Sarcoma }} & \multirow{2}{*}{$\begin{array}{l}\text { Number of } \\
\text { Cases } \\
\text { Treated }\end{array}$} & \multirow{2}{*}{ Age (yrs) } & \multicolumn{2}{|c|}{ Sex } & \multicolumn{3}{|c|}{ Side } & \multicolumn{2}{|c|}{ Survival (yrs) } \\
\hline & & & & $\mathbf{M}$ & $F$ & $\mathbf{R}$ & $\mathrm{L}$ & B & 5 & 3 \\
\hline Lymphoma & $\ldots$ & 6 & $\begin{array}{c}40-79 \\
\text { (average 53) }\end{array}$ & 3 & 3 & 0 & 4 & 2 & $1 / 2$ & $5 / 5$ \\
\hline Lymphosarcoma & $\ldots$ & 7 & $\begin{array}{c}47-82 \\
\text { (average 64) }\end{array}$ & 4 & 3 & 2 & 4 & 0 & $3 / 3$ & $4 / 6$ \\
\hline Reticulosarcoma & $\ldots$ & 1 & 53 & $M$ & 0 & 1 & 0 & 0 & \multicolumn{2}{|c|}{ Alive 13 yrs } \\
\hline
\end{tabular}

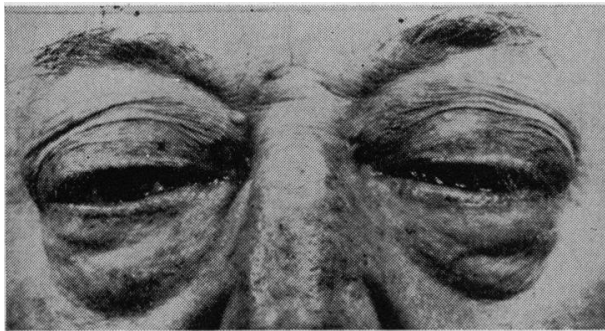

(A) Before radiation treatment.

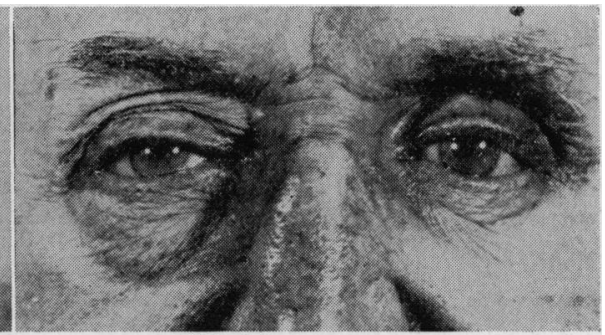

(B) After treatment.

This patient developed a leukaemic blood picture and died of dissemination 2 years later.

FIG. 4.-Bilateral orbital lymphoma.

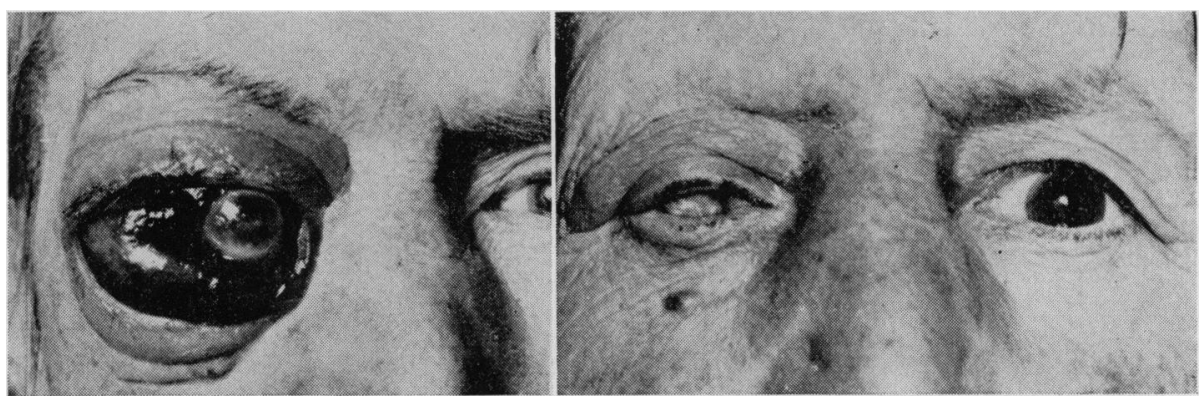

(A) Before radiation treatment.

(B) After treatment.

The eye was not removed, and the patient died of a local recurrence 5 years later.

FIG. 5.-Lymphosarcoma of orbit.

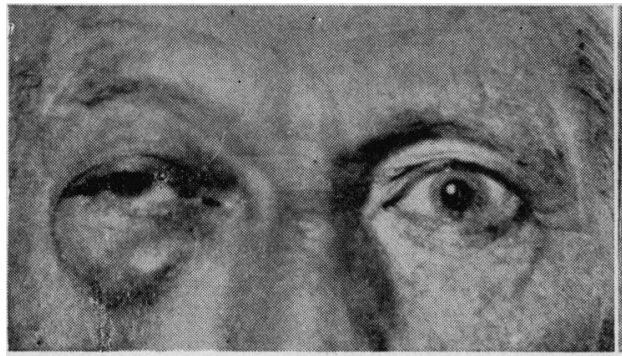

(A) Before radiation treatment.

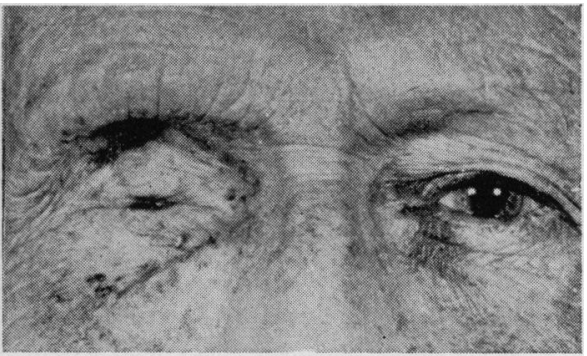

(B) 13 years after treatment.

The patient was monocular. Blind right eye removed before starting radium beam therapy. Fig. 6.-Reticulosarcoma. 
Rhabdomyosarcomata.-Our experience of this rare tumour was limited to seven orbital and four extra-orbital cases. Histological sections were available for all the orbital cases; in three the presence of striations was demonstrated, and in the rest the clinical features, course of disease, and response to radiation justified the diagnosis.

It is generally agreed that these tumours are exceedingly rare, occur in children, and are highly lethal. Reese (1951) notes 25 cases of undisputed orbital rhabdomyosarcoma in the literature and reports two 10-year survivals out of five of his own patients treated by exenteration. He states categorically that the tumours are radio-resistant and this view is supported by Duke-Elder (1952). Offret (1951) repeats the accepted view concerning radio-sensitivity and points out these tumours may show variations in rate of growth, and advances Masson's unsupported hypothesis that there are two forms of rhabdomyosarcoma: a typical form of congenital origin, and an atypical form of acquired origin. In a subsequent chapter, however, under the heading "malignant reticular tumours", Offret gives a classical account of the behaviour and response of the tumours paralleled in our series by the "rhabdomyosarcomata". The experience obtained in the radiation treatment of our series of cases leads us to believe that they occur mostly in children, remain localized to the orbit, and are highly radio-sensitive. They are, however, only exceptionally radio-curable, since prompt recurrence is nearly always the rule (Fig. 7).

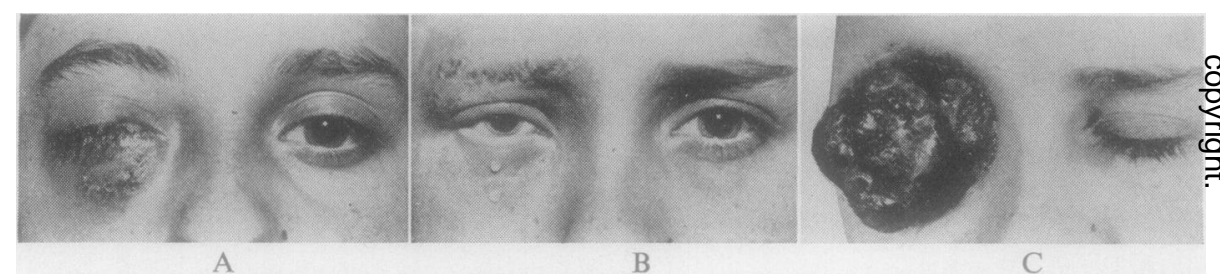

(A) Before radiation treatment.

(B) Regression with immediate recurrence after treatment

(C) Recurrence in orbit immediately after exenteration.

FIG. 7.-Rhabdomyosarcoma of orbit.

Only the two eldest patients in this series have survived, and one of them has developed xerophthalmia and has little vision in the eye (Fig. 8).

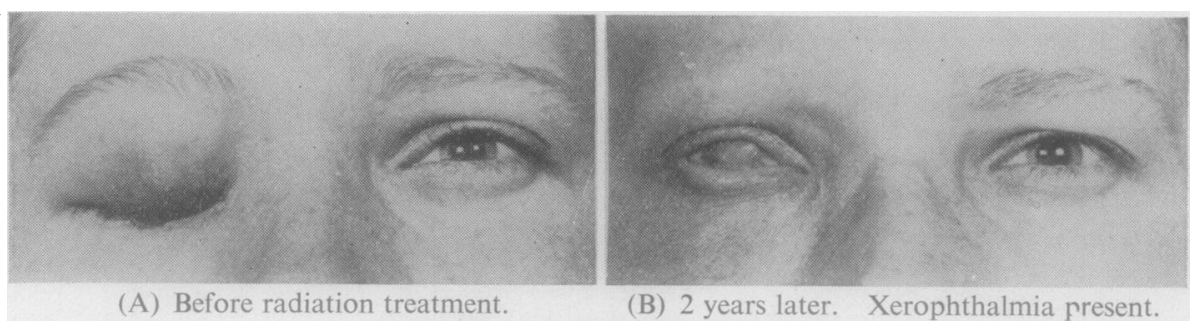

FIG. 8.-Rhabdomyosarcoma of orbit.

The longest survivor has evidence of a radiation cataract (Fig. 9 opposite), and the third survivor, aged 6, has so far remained free of recurrence for 6 months (Table V, opposite). 


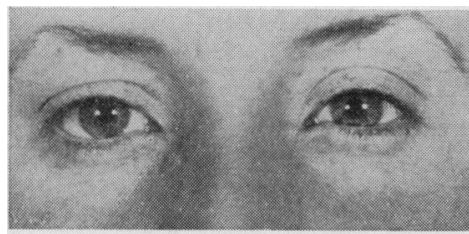

A

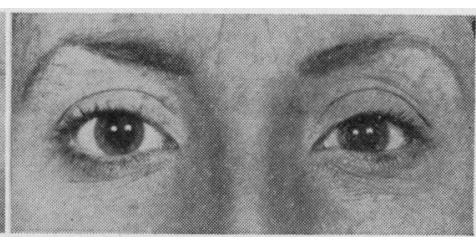

B

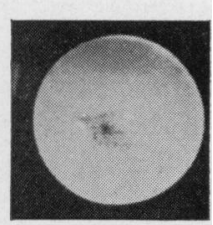

$\mathrm{C}$

FIG. 9.-Rhabdomyosarcoma of orbit. (A) Before radiation treatment. (B) 3 years after treatment. (C) Cataract occurring within 39 to 42 months of treat ment.

TABLE V

RHABDOMYOSARCOMA

\begin{tabular}{|c|c|}
\hline Number of Cases & 7 \\
\hline Age Range (yrs) & $3-26$ \\
\hline Sex & 2 Male, 5 Female \\
\hline Side & 5 Right, 2 Left \\
\hline Histologically Proven & $\begin{array}{l}3 \text { Rhabdomyosarcoma } \\
3 \text { Sarcoma, suggestive of rhabdomyosarcoma } \\
1 \text { Undifferentiated sarcoma }\end{array}$ \\
\hline Survivors & $\begin{array}{l}1 \text { for } 3 \text { yrs (aet 19)-proven } \\
1 \text { for } 1 \frac{1}{2} \text { yrs (aet 26)- - suggestive } \\
1 \text { for } 3 \text { mths (aet 6)-undifferentiated sarcoma }\end{array}$ \\
\hline
\end{tabular}

The rhabdomyosarcoma (or, when the typical cells cannot be demonstrated, the round cell, mixed cell, embryonic, or anaplastic tumour, to use some of the histological terms employed) should always be treated by radiotherapy in the first instance. All these tumours have so far disappeared under radiation, though many have recurred promptly in which event exenteration has been performed. There is no evidence to show that immediate exenteration has any advantage for cases behaving in this fashion and, as these tumours appear to remain localized, any delay in operation because of the preliminary attempt at cure by radiotherapy is not apparently associated with an increased risk of metastases. In the four patients who died of this disease, distant metastases were not demonstrated, although post-mortem examinations were not done. Distant metastases did occur, however, in the extra-orbital group which also proved less responsive to radiation.

Lacrimal Gland Tumours.-These are rare, the commonest being the "mixed" tumour which appears to be analogous both clinically and histologically to the mixed tumour affecting the major and minor salivary glands of the buccal cavity and pharynx. These tumours have a common epithelial origin but because of their cellular pleomorphism the histological appearances may range from the benign adenoma to the adenocarcinoma, and a tumour may sometimes be completely anaplastic. These wide variations in morphology are reflected in their clinical behaviour, which may vary from apparent simplicity to high malignancy with widespread metastases. However benign the histological picture, these tumours should always be regarded clinically as malignant.

The mixed or pleomorphic tumours of the salivary and lacrimal glands form the largest group of tumours affecting these organs: not only is their site of origin 
singular, but they are unique among human neoplasms in their natural history. No other epithelial neoplasm equals the mixed tumour in its characteristic slow remorseless progress: cure by any method of treatment cannot be judged in terms of the ordinary 3- or 5-year survival rates but rather by decades, and even then after half a lifetime the patient is never free from the risk of recrudescence.

Death from metastases is unusual but may occur early in the course of the disease where the primary tumour is a rapidly-growing undifferentiated adenocarcinoma or a cylindroma. With the so-called mixed tumours of the lacrimal gland, the tempo of recurrence and spread is usually more rapid than with the corresponding tumours of the main salivary glands and the prognosis is therefore much graver. Reese (1951) attributes the high proportion of recurrences and the high mortality from lacrimal gland tumours to the difficulties of surgical approach and removal, the tendency to invasion of the capsule, and particularly the early invasion of bone (Fig. 10).

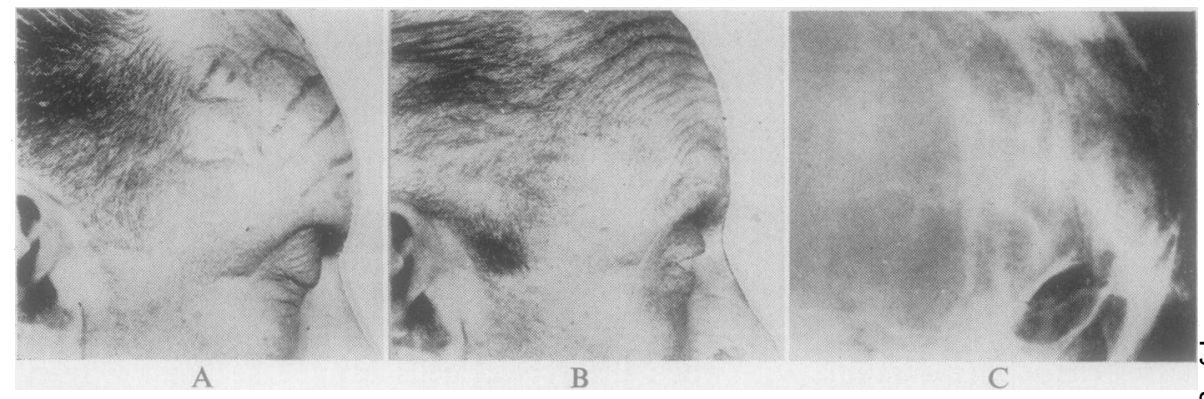

(A) Before radiation treatment. (B) After treatment. (C) Skiagram of same case before treatment Fig. 10.-Lacrimal gland tumour showing invasion of skull.

It is a common observation that mixed salivary tumours are relatively radioresistant. I have treated a large number of salivary gland tumours by radiation pre-operatively and have also had abundant opportunity of treating post-operative recurrences. Radiotherapy can help reduce the recurrence rate within the limitations and fallacies imposed by the 5-year period of observation, and should therefore be routinely used in association with surgery. Radiotherapy is, of course, the method of choice for the inoperable, recurrent or highly malignant epithelial tumours and the lymphosarcomata. Table VI (opposite) shows the results obtained.

Two cases of primary adenocarcinoma of the lacrimal gland survived for over 10 years, one patient retaining a normal eye (Fig. 11).

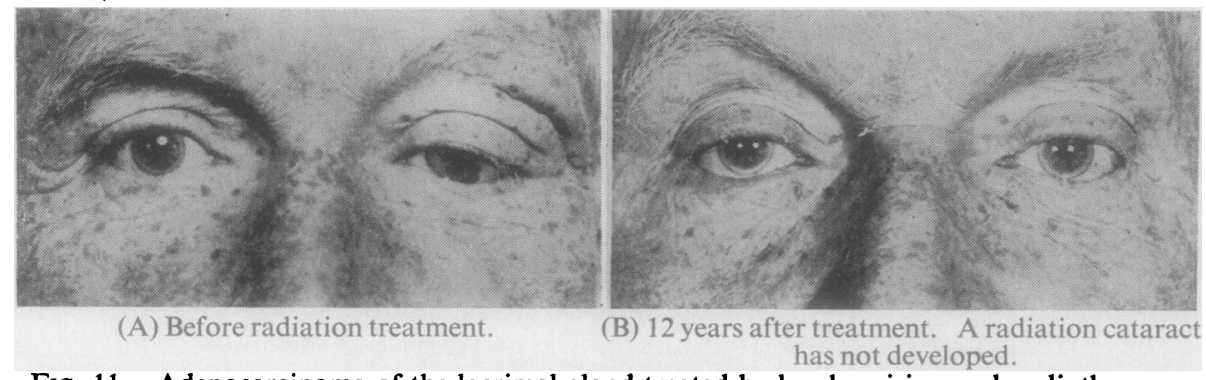

Fig. 11.-Adenocarcinoma of the lacrimal gland treated by local excision and radiotherapy. 
TABLE VI

LACRIMAL GLAND TUMOURS

\begin{tabular}{|c|c|c|c|c|c|c|c|c|c|c|c|}
\hline \multirow{3}{*}{ Type of Tumour } & \multirow{3}{*}{$\begin{array}{l}\text { No. } \\
\text { of } \\
\text { Cases }\end{array}$} & \multirow{3}{*}{ Age (yrs) } & \multicolumn{2}{|c|}{ Sex } & \multicolumn{3}{|c|}{ Side } & \multicolumn{2}{|c|}{ Removal of Eye } & \multirow{2}{*}{\multicolumn{2}{|c|}{$\underset{\text { (yrs) }}{\text { Survival }}$}} \\
\hline & & & & & & & & & & & \\
\hline & & & $\mathbf{M}$ & $\mathbf{F}$ & $\mathbf{R}$ & $\mathbf{L}$ & B & radiation & radiation & 5 & 3 \\
\hline Mixed & 5 & $\begin{array}{c}37-76 \\
\text { (average 53) }\end{array}$ & 3 & 2 & 2 & 3 & 0 & 2 & - & $2 / 3$ & $3 / 4$ \\
\hline Adenocarcinoma $\ldots$ & 4 & $\begin{array}{c}36-52 \\
\text { (average 46) }\end{array}$ & 1 & 3 & 0 & 4 & 0 & 3 & - & $2 / 3$ & $3 / 4$ \\
\hline $\begin{array}{c}\text { Undifferentiated } \\
\text { Carcinoma }\end{array}$ & 3 & $\begin{array}{c}31-65 \\
\text { (average 43) }\end{array}$ & 1 & 2 & 1 & 2 & 0 & 1 & - & $1 / 2$ & $1 / 2$ \\
\hline Lymphosarcoma $\ldots$ & 2 & $\begin{array}{c}49 \text { and } 59 \\
\text { (average 54) }\end{array}$ & 1 & 1 & 0 & 1 & 1 & - & - & $0 / 1$ & $1 / 2$ \\
\hline
\end{tabular}

In the one patient with undifferentiated carcinoma who survived for 5 years, the tumour arose primarily in the lacrimal sac; it is included here for convenience.

Other Tumours.-Table VII lists the results obtained and warrants no special comment.

TABLE VII

OTHER MALIGNANT TUMOURS

\begin{tabular}{l|c|l}
\hline \multicolumn{1}{c|}{ Histology } & No. of Cases & \multicolumn{1}{|c}{ Result } \\
\hline Liposarcoma & 1 & Died of disease after 5 yrs \\
\hline Mixed cell sarcoma & 1 & Died of disease after 4 yrs \\
\hline Glioma optic nerve & 1 & Alive after 5 yrs \\
\hline Various sarcomata & 2 & Died of disease \\
\hline No pathology & 1 & Died of disease after $2 \mathrm{mths}$ \\
\hline
\end{tabular}

\section{Secondary Malignant Tumours (Table VIII, overleaf)}

Intra-Ocular Tumours.-A malignant intra-ocular tumour is usually treated by removal of the affected eye. Radiotherapy is reserved as a post-operative measure if examination of the excised eye shows evidence of extra-ocular spread, or if frank post-operative recurrence be detected in the socket or orbital cavity after exenteration. In only three of a series of 31 choroidal melanomata irradiated postoperatively were frank orbital deposits visible (Fig. 12, overleaf).

In the case of the retinoblastoma, irradiation is reserved either for neoplasm occurring in the sole remaining eye, or as a post-operative measure if extra-ocular extension be detected or if frank recurrence takes place in the socket. There were three patients in the last category, out of a total of twenty seen with retinoblastomata (Fig. 13, overleaf). 
TABLE VIII SECONDARY MALIGNANT TUMOURS

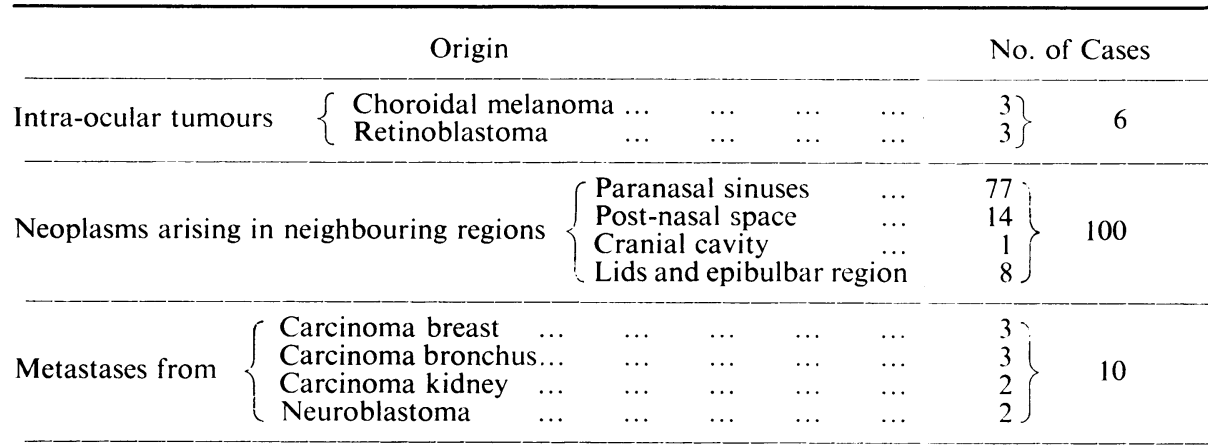

\begin{tabular}{|c|c|c|}
\hline Orbital manifestations of generalized lymphoid tumours & $\ldots$ & 10 \\
\hline
\end{tabular}

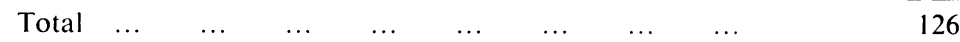

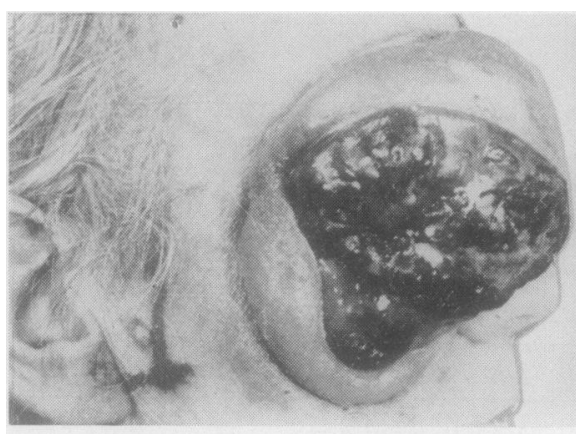

(A) Before radiation treatment.

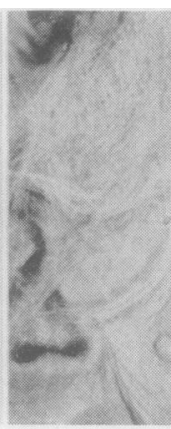

(B) After treatment.

FIG. 12.-Intra-ocular melanoma.

(Reproduced by courtesy of the Editor of the British Journal of Radiology).

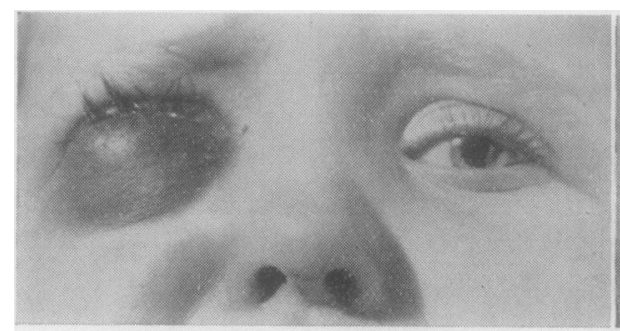

(A) Before radiation treatment.

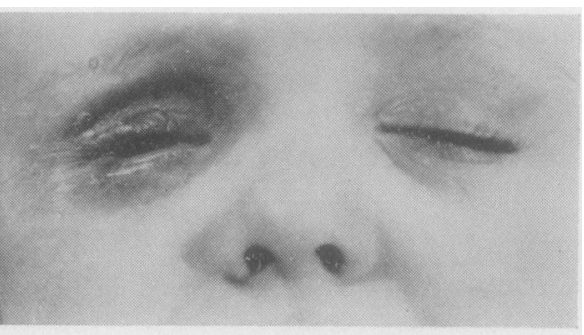

(B) After treatment.

FIG. 13.-Retinoblastoma: post-operative recurrence.

Metastatic Tumours (Figs 14, 15, and 16, opposite).-These are rare and usually comprise one dramatic manifestation of a generalized dissemination. An orbital metastasis should be treated by radiotherapy if the patient's general condition is satisfactory; otherwise the patient may live long enough to develop the distressing symptoms associated with progressive and uncontrolled proptosis and his $\stackrel{?}{+}$ 


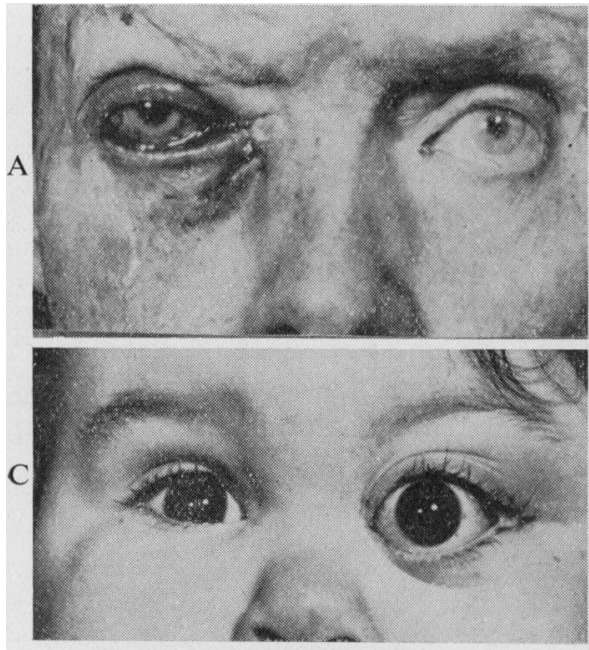

(A) From carcinoma of the breast.

(C) From neuroblastoma.
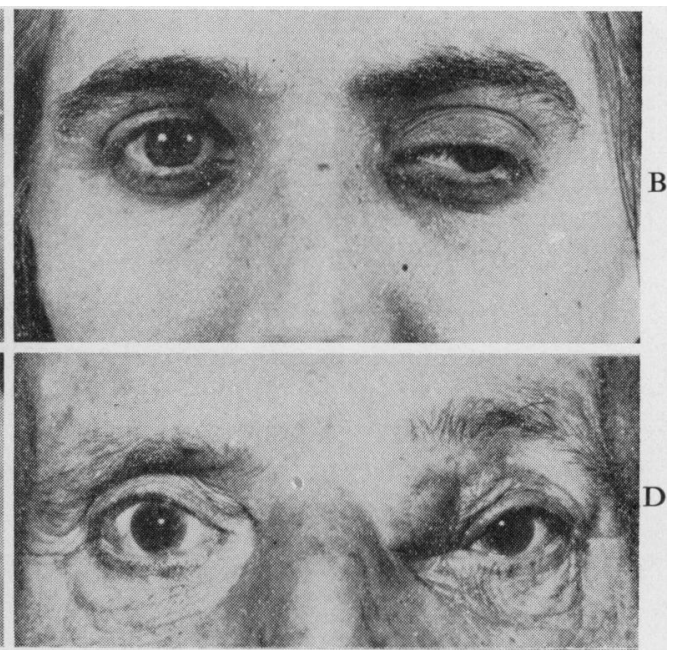

(B) From hypernephroma.

(D) From carcinoma of the bronchus.

FIG. 14.-Secondary deposits in the orbit.

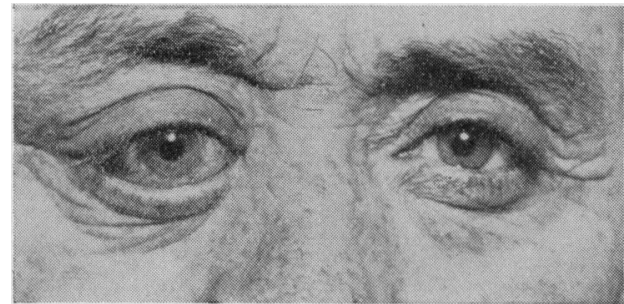

(A) Before radiation treatment.

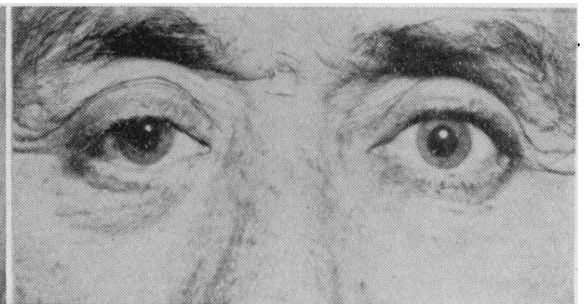

(B) After treatment.

Fig. 15.-Secondary deposit in the orbit from carcinoma of the bronchus.

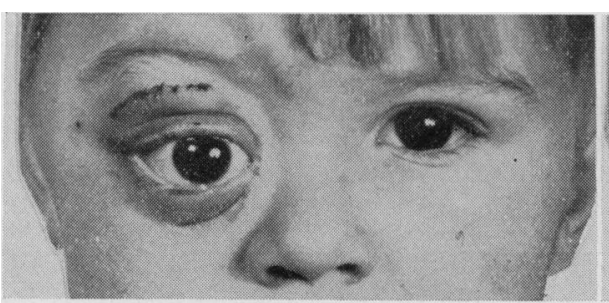

(A) Before radiation treatment.

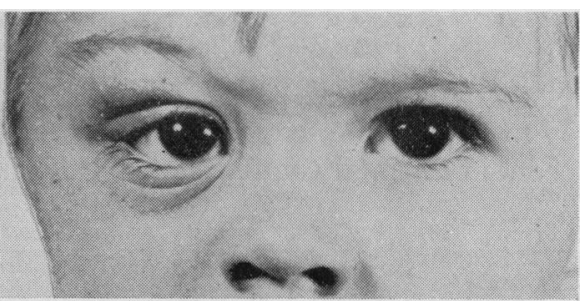

(B) After treatment.

Fig. 16.-Orbital neuroblastoma.

terminal state become truly wretched. Secondary deposits of this type respond reasonably well to radiotherapy, and these patients can usually be spared removal of the eye or more radical orbital surgery.

Tumours of the Lids and Epibulbar Region.--Secondary invasion of the orbit from cancer of the lids was not uncommon in the pre-radiation era. It is rare nowadays 
to meet a rodent ulcer which has either been so neglected or so failed to be controlled by treatment that progress to the stage of orbital invasion and destruction of the orbital contents has occurred. Extension to the orbit from primary cancers of the lid and epibulbar region should be treated in the first instance by radiotherapy, and this should be followed by radical surgery whenever possible. Radiotherapy alone is usually of palliative value only, and surgery without radiotherapy is likely to be followed by recurrence.

Generalized Lymphoid Tumours (Fig. 17).-Orbital lesions occurring during the course of an already established and generalized lymphoid tumour are not common (1.7 per cent. of cases: Duke-Elder, 1952). There were ten cases in a series of some three hundred patients seen at the Royal Marsden Hospital from 1945-1955. Radiation is the treatment of choice.

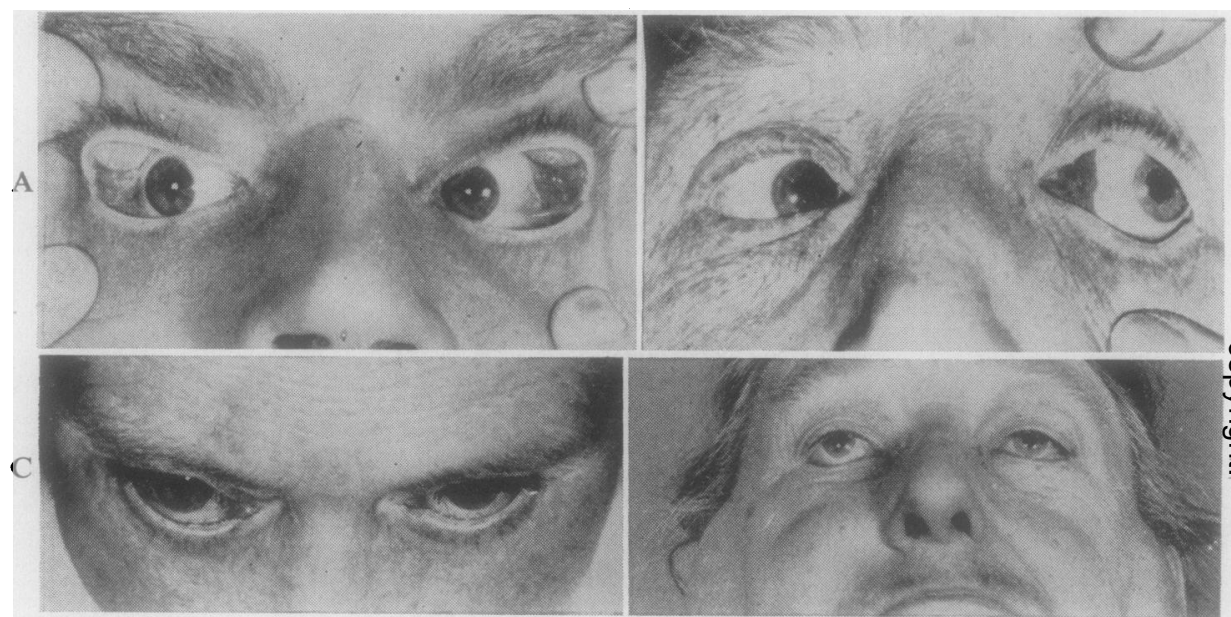

(A) Lymphosarcomatous deposits in lacrimal glands.

(B) Leukaemic deposit in orbit and conjunctiva.

(C) Orbital deposit in Brill-Symmers disease.

(D) Mikulicz syndrome in lymphosarcoma.

FIG. 17.-Orbital manifestations of generalized lymphoid tumours.

Invasion from Sinuses and Post-Nasal Space (Figs 18 and 19).-The commonest secondary orbital tumour seen by the radiotherapist follows invasion of the orbit

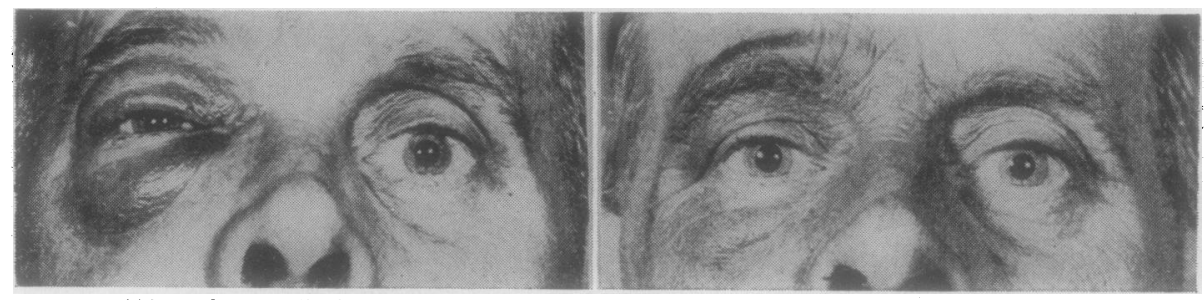

(A) Before radiation treatment.

(B) After treatment.

Fig. 18.-Maxillo-ethmoidal carcinoma. 


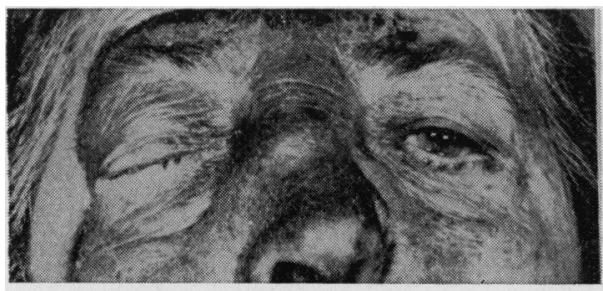

(A) Before radiation treatment.

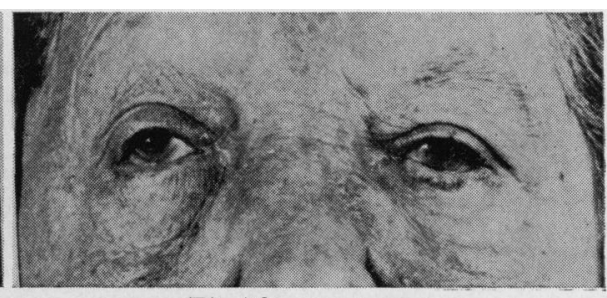

(B) After treatment.

FIG. 19.-Ethmoidal carcinoma.

from a paranasal sinus cancer; or from the post-nasal space. Table IX shows the incidence of orbital invasion in a series of cases seen at the Royal Marsden Hospital. This process is always of grave significance as far as prognosis is concerned and adds considerably to the technical difficulties of treatment because of the need to avoid radiation damage to the eye.

TABLE IX

CANCER OF PARANASAL SINUSES AND NASOPHARYNX

\begin{tabular}{llll|c|c|c}
\hline Site of Carcinoma & $\cdots$ & $\ldots$ & $\ldots$ & Antrum & Ethmoid & Nasopharynx \\
\hline Orbital invasion & $\ldots$ & $\ldots$ & $\ldots$ & 54 & 23 & 14 \\
\hline Cranial nerve involvement & $\ldots$ & $\ldots$ & 17 & 5 & 43 \\
\hline Post-radiation complications... & $\ldots$ & 15 & 4 & 5 \\
\hline Eye removed $\ldots$ & $\ldots$ & $\ldots$ & $\ldots$ & 5 & 3 & - \\
\hline Orbit exenterated & $\ldots$ & $\ldots$ & $\ldots$ & 6 & 3 & 165 \\
\hline
\end{tabular}

Removal of a sound eye or exenteration of the orbit in the treatment of antral or ethmoidal cancer is never justifiable as a primary measure, for it adds little to the patient's chances of survival. The presence of neoplasm in the orbit is an index of the extensive nature of the disease and if radiotherapy is first applied and fails then subsequent surgery is no more likely to be successful. In our total series of 172 cases of antral and ethmoidal carcinoma there were nine orbital exenterations and only one patient was still alive a year later. Of the eight other patients who lost the eye (in no case was removal necessitated by radiation damage) two have lived for more than 3 years. In both cases the patients came into the hands of plastic surgeons; one eye was removed because of infection and the other in spite of advice to the contrary (Table IX).

\section{Complications and Sequelae of Radiotherapy}

In advocating radiotherapy for the treatment of malignant orbital tumours one must be ever conscious of the possible risks of damage to the normal eye. If the risks of radiation damage to the eye are considerable, then a case can be made out for removing the eye before embarking upon radiotherapy. If, however, the risks can be shown to be small or otherwise worth accepting, 
then clearly the eye should be left, since it is far better for a patient to retain the eye even at the cost of impaired vision than suffer its removal, which is $\frac{\vec{J}}{3}$ inevitable when a malignant orbital tumour is submitted to surgical treat- $\stackrel{\circ}{-}$ ment.

The sinister reputation of radiotherapy among ophthalmologists is well $\frac{\vec{\rho}}{0}$ known. Under modern conditions the ocular disasters encountered in the $\frac{0}{6}$ early days are rarely seen, though it would be wrong to give the impression $\frac{\overrightarrow{\vec{p}}}{\vec{\sigma}}$ that the irradiation of a malignant orbital tumour is without risk. Complete $\stackrel{\odot}{\circ}$ protection of the eye cannot be obtained nor is it desirable since tumour $\stackrel{ }{\%}$ tissue may simultaneously be protected; the most one can hope to achieve is $\vec{\circ}$ to protect from direct radiation the vulnerable ocular tissues forming the $\vec{\omega}$ anterior segment of the eye, namely the cornea, iris, ciliary body, and lens.

The risks of radiation damage to the eye depend on the following factors:

(1) The radio-sensitivity of the tumour and the dosage employed.

(2) The state of the eye before treatment.

(3) The technical method of radiation employed.

(4) The degree of protection possible.

(1) Radio-sensitivity and Dosage.-There is a material variation in the radio- $\vec{\circ}$ sensitivity of various parts of the normal eye. Thus the outer eye, comprising $\stackrel{\circ}{\circ}$ the lids, conjunctiva, and cornea, tolerates radiation well and any reactions occur- $\Phi$ ring usually heal without serious or permanent damage (Fig. 20, opposite). The $\vec{\varphi}$ normal cornea tolerates radiation even when high doses are given-providing o sepsis and trauma are avoided. Of the intra-ocular structures, the lens alone highly radio-sensitive, a single dose of 600r or 1,500r in 1 month being almo certain to produce a cataract. Little is known concerning the radio-sensitivity of the retina and uveal tract.

Tumour sensitivity and the dosage of radiation required vary considerably. $\frac{0}{2}$ The lymphoid sarcomata are radio-sensitive and a dose of 2,000-3,000r delivered $\overrightarrow{\overrightarrow{0}}$ during one month proved adequate for most cases, but secondary orbital invasion $\rightrightarrows$ from cancer of the paranasal sinuses may prove most obstinate and high doses of $5,000-6,000$ r in 4-6 weeks often prove inadequate, especially with extensive bone involvement. Secondary deposits from other primary tumours or spread from $\frac{0}{0}$ intra-ocular tumours can usually be controlled by doses of about 4,500 in 1 month. $\stackrel{\circ}{\circ}$ The secondary manifestations of the reticuloses are as sensitive as the lymphoid sarcomata or more so.

The techniques employed for treating patients suffering from benign orbital tumours (usually haemangioma) should be risk-free as far as the eye is concerned. $\bigcirc$ The dosage and timing of the treatment should be such that visible reactions are $\rightarrow$ avoided and the eye completely protected from direct radiation.

(2) State of the Eye before Radiation Treatment.-This plays a large part in $\stackrel{\sim}{\circ}$ determining whether or not an ocular complication will develop. When the eye is $\tilde{N}$ normal in position and the lids are normal, there is less likelihood of damage than $\underset{\mathcal{W}}{\mathcal{N}}$ when the eye shows a severe degree of proptosis with chemosis of the conjunctiva 2

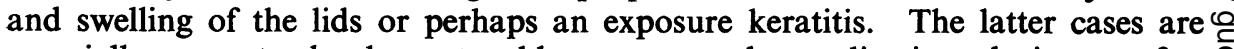

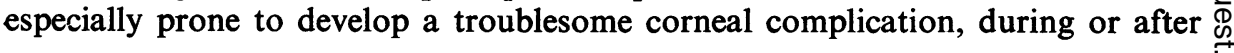




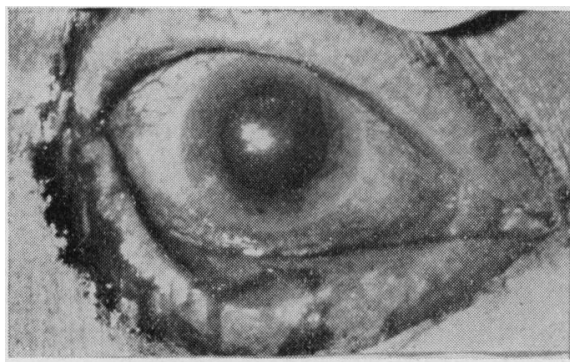

(A) Eye during stage of full reaction.

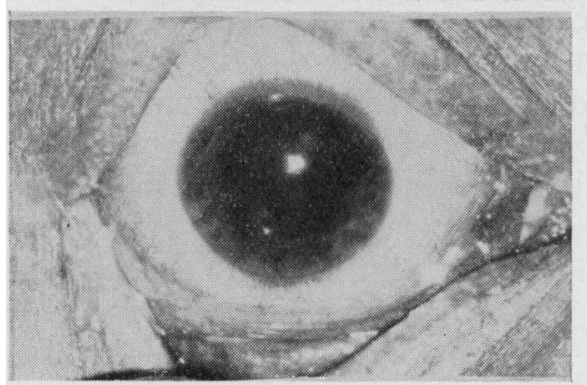

(B) 18 days later-corneal oedema with superficial punctate staining.

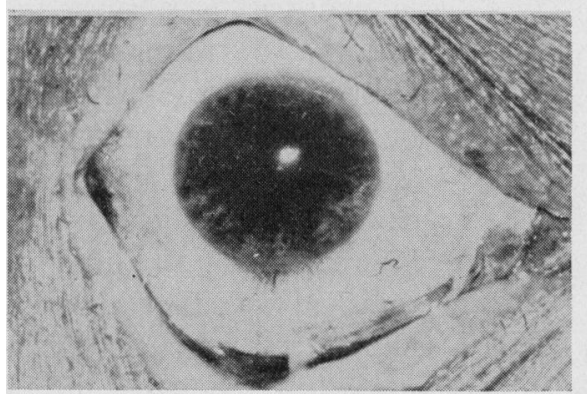

(C) 8 months later-reaction subsided.

FIG. 20.- Reaction of normal eye to teleradium therapy $(12 \mathrm{~g}$. unit). The eye was irradiated directly without any protection.

radiotherapy. Visual acuity which has become reduced because of the presence of an orbital tumour is not generally improved after successful radiation treatment; the most one can hope for is the prevention of further deterioration of vision as the tumour regresses and the eye returns to a more normal position.

(3) Technical Method.-This is of fundamental importance since the close application of radio-active sources, i.e. radon seeds, radium needles, tantalum wire rings, all carry a high risk of producing marked changes in the lens, conjunctiva, and cornea, some of which may be permanent. External radiotherapy is the ideal method of treatment for an orbital tumour, local methods of application being reserved for cases in which the eye has already been removed. Of the external methods of radiation available, high-voltage or supervoltage $x$-rays are preferable to teleradium or telecobalt therapy, in which efficient ocular protection is almost impossible.

(4) Degree of Ocular Protection.-This depends on the nature and extent of the tumour and region of the orbit affected. Most primary malignant orbital tumours demand the irradiation of the whole orbit and an important fraction of the eye, as otherwise one cannot be certain that all the tumour tissue will be irradiated. 
However, in the case of a palpable localized tumour of epithelial origin, such as a lacrimal gland tumour or an orbital invasion secondary to paranasal sinus cancer, $\frac{\omega}{\overline{3}}$ radiation can be restricted to the affected portion of the orbit. Where the whole $\frac{\text { o }}{\text {. }}$ orbit has to be irradiated, the cornea and the intra-ocular structures deep to it can $\underset{\overrightarrow{\vec{s}}}{\overrightarrow{\vec{p}}}$ be shielded from direct radiation to a variable degree. By this means a keratitis and iridocyclitis may be avoided, but a cataract may still subsequently develop $\frac{\sigma}{\sigma}$ because of the amount of scattered radiation reaching the lens.

Table $\mathrm{X}$ lists the chief ocular complications encountered. These are $\stackrel{\overparen{D}}{\stackrel{D}{2}}$ permanent lesions due to damage by radiation and are to be carefully distinguished from temporary reversible changes which are generally termed $\overrightarrow{0}$ the "reaction". The incidence of certain complications is not quoted in $\overrightarrow{\vec{\omega}}$ Table $\mathrm{X}$ because these must be accepted as foreseeable and calculated risks.

TABLE $X$

COMPLICATIONS OF RADIOTHERAPY

\begin{tabular}{|c|c|c|c|}
\hline \multirow{2}{*}{\multicolumn{2}{|c|}{ Complication }} & Orbital & Tumour \\
\hline & & Primary & Secondary \\
\hline $\begin{array}{l}\text { Epilation } \ldots \\
\text { Lid scarring and deformity } \\
\text { Socket contraction ... } \ldots\end{array}$ & $\begin{array}{l}\cdots \\
\cdots \\
\cdots\end{array}$ & $\overline{-}$ & $\bar{z}$ \\
\hline $\begin{array}{l}\text { Chronic conjunctivitis } \\
\text { Xerophthalmia } \quad \ldots\end{array}$ & $\begin{array}{l}\cdots \\
\cdots\end{array}$ & $\overline{1}$ & - \\
\hline $\begin{array}{l}\text { Superficial punctate keratitis } \\
\text { Corneal ulcer } \\
\text { Corneal vascularized opacity }\end{array}$ & $\begin{array}{l}\cdots \\
\cdots \\
\cdots\end{array}$ & $\begin{array}{r}4 \\
2 \\
-\end{array}$ & $\begin{array}{l}3 \\
4 \\
1\end{array}$ \\
\hline Vitreous haemorrhages $\quad \ldots$ & $\ldots$ & 1 & 1 \\
\hline Cataract $\quad \ldots$ & $\cdots$ & $\begin{array}{l}6 \text { single } \\
1 \text { bilateral }\end{array}$ & $\begin{array}{l}14 \text { single } \\
5 \text { bilateral }\end{array}$ \\
\hline Osteonecrosis & $\ldots$ & - & - \\
\hline
\end{tabular}

Thus epilation of the browo and lashes can in a given case be predicted with cer- $\dot{0}$ tainty and no attempt can ${ }_{0}$ be made to avoid this type ${ }^{N}$ of damage. Similarly, osteonecrosis is almost in- $\mathrm{O}$ evitable if radiation be $\frac{0}{0}$ applied to bone which is $\stackrel{\Phi}{\stackrel{\Phi}{\sim}}$ both invaded by neoplasm $\overrightarrow{0}$ and infected; in sone on cases this complication ca be avoided by preliminary removal of the invaded and infected bone. Post-

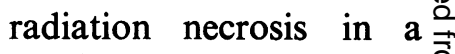
previously normal bone is exceptional.

Radiation Cataract.-This is by far the commonest single complication and is to be expected in nearly all malignant cases treated. So far we have encountered twenty unilateral and six bilateral cataracts. There is usually a latent period of some 3 years between the treatment and the onset of the cataract, which as a rule occasions surprisingly little complaint. Extraction of the cataract has been performed in two cases, apparently without undue difficulty. It is justifiable to run the risk of radiation cataract in treating a malignant lesion of the eye or orbit where no alternative to radiotherapy exists or where surgical treatment would entail enucleation.

Superficial Punctate Keratitis.-This can be a troublesome sequel to the keratitis which occurs if the eye is not protected. It may also develop when the cornea is directly protected, and may then be ascribed to secondary irritation of the cornea from the reaction in the lids.

Corneal Ulceration.-This can rarely be directly attributed to radiation, since in 
the absence of trauma or infection the cornea tolerates therapeutic dosage well, but it is sometimes encountered in the following circumstances:

(1) After contact or direct radiation where the radiating source is placed on or near the cornea,

(2) As part of an exposure keratitis consequent upon the uncontrolled progress of the orbital disease.

(3) In the form of superficial ulceration of the lower part of the cornea followed later by a vascularized scar.

This vascularized scarring of the cornea is very rare and characteristically affects the lower half or third of the cornea. One case of this kind was seen in this series, and another occurred after irradiation of a lower-lid tumour. In both these cases, marked post-radiation changes were present in the lower lid on the affected side (Fig. 21).

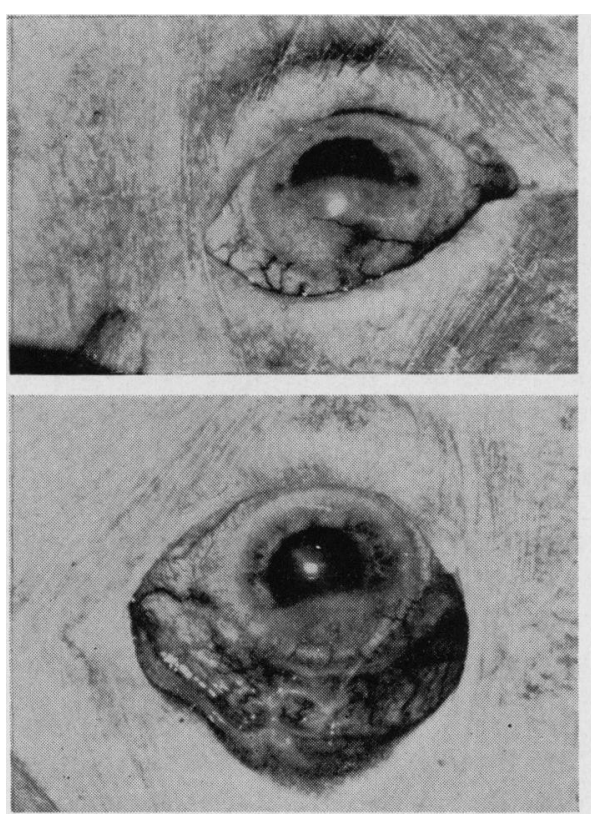

(A) After irradiation of carcinoma of the antrum (teleradium therapy without ocular protection).

(B) After irradiation of a lid tumour ( $x$-ray therapy with protection of the eye).

FIG. 21.-Vascularized corneal opacity. In both cases the lower lid shows permanent radiation changes.

Reese (1951) attributes this type of corneal change to the process of keratinization of the corneal and conjunctival epithelium which may follow radiotherapy. This may well be so, but the alternative possibility that the corneal lesion is secondary to the associated lower-lid changes must also be considered in view of the rarity of the complication.

Lacrimal Secretion.-Irradiation of the major salivary glands of the mouth is followed by alterations in the quality and quantity of the secretion of saliva. Such changes, which result chiefly in dryness of the mouth associated with a much diminished and viscid saliva, can be permanent; the affected mucous membranes become dry and glazed and the teeth if present become carious. Similar changes 
in the quality and quantity of the tears secreted may cause a chronic irritable eye or a xerophthalmia (Fig. 8).

Reese ascribes the chronic irritable eye to keratinization, keratin being des- $\frac{\mathrm{O}}{-}$ quamated into the conjunctival sac. Fortunately, irradiation is rarely admini- $\overrightarrow{\vec{F}}$ stered to the lacrimal and associated glands in sufficient quantity to damage their secretory function permanently or severely.

Vitreous Haemorrhage.-This is an exceptional complication. Reese en- $\frac{\vec{p}}{\overrightarrow{0}}$ countered it after treating intra-ocular tumours (retinoblastoma) by his special $D$ technique of $x$-ray therapy, whereby a high dose of radiation, $8,000 \mathrm{r} \times 2$, is given is over $3 \frac{1}{2}$ months using $220 \mathrm{kV} x$-rays; 34.5 per cent. of the retinoblastomata so $\overrightarrow{0}$

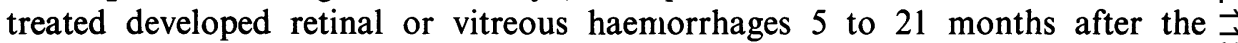
completion of treatment.

If at all frequent a serious vascular complication of this kind, which may result $\frac{\partial}{0}$ in loss of vision, might well make radiotherapy pointless since the possibility of saving vision is its chief advantage over surgery. Fortunately, in treating an orbital as opposed to an intra-ocular tumour, the risk of retinal or vitreous haemor- of rhage is apparently negligible. Only two patients developed this complication $\stackrel{0}{N}$ and in neither was it certain that the radiation was the cause of the haemorrhage. 9

In the first case, a patient suffering from a carcinoma of the antrum 9 years after treat- $\vec{P}$ ment developed vitreous haemorrhage associated with a choroido-retinal degeneration $\bigcirc$ over the region of the eye related to the diseased part of the orbit. Removal of the floor $\vec{O}$ of the orbit had been undertaken for osteonecrosis several years before. It is not im- $\stackrel{\Phi}{工}$ possible that the localized changes in the eye were due to surgical trauma, since the ocular $\vec{\varphi}$ appearances were not unlike those which follow the application of diathermy.

In the second case, a tumour of the lacrimal gland was treated post-operatively b $x$-ray therapy, the eye being shielded. A vitreous haemorrhage developed 2 years late and has since recurred. The patient retains some vision but unfortunately has only the one eye. Apart from epilation there are no other stigmata of radiation change or damage.

Vitreous haemorrhage without radiation cataract may not be due to radiotherapy, for retinal or vitreous haemorrhage is an expression of a damaged vasculature, and the high dosage of radiation which would produce the requisite degree of damage would certainly be high enough to produce a cataract.

In the treatment technique used by the writer, the retinal vessels are irradiated, but the dosage and technique are fundamentally different from those employed by Reese in treating retinoblastoma; his method is more likely to be followed by marked post-radiation changes in the retinal blood vessels than more conventional methods using a lower dosage in a shorter time. When techniques comparable to those used by Reese for retinoblastoma are used for carcinoma of the head, neck, and breast, marked connective tissue changes including sclerosis of blood vessels are known to occur.

Epilation of the Lashes and Brows.-This is cosmetically unwelcome but never serious. The lower lashes usually suffer first, to be followed by the upper lashes and finally by the brow. Epilation of the eyebrow is more likely to be permanent than epilation of the lashes.

Scarring.-The permanently epilated lid has a tendency to become keratinized and deformed. 
Socket Contraction (Fig. 22).-This is inevitable after external radiation or heavy local irradiation. The process of fibrosis and contraction begins immediately after the reaction subsides; it progresses rapidly to the state where a prosthesis cannot be accommodated, and ultimately the socket shrinks up completely, the epithelium undergoes metaplasia, and all that remains is a shallow trough-like depression between two hairless and atrophic lid margins. This complication is unsightly and difficult to remedy once it has become established, but

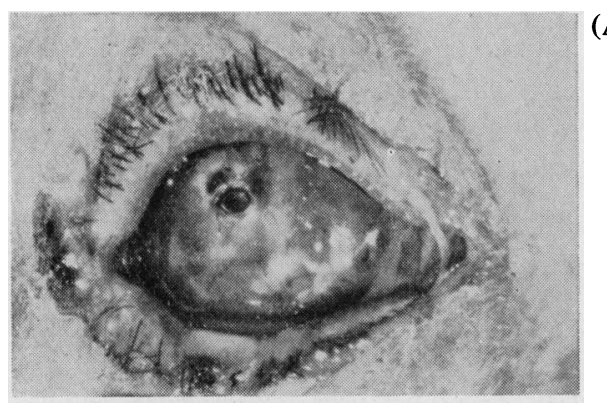

(A) Socket showing reaction and glass shell in place.

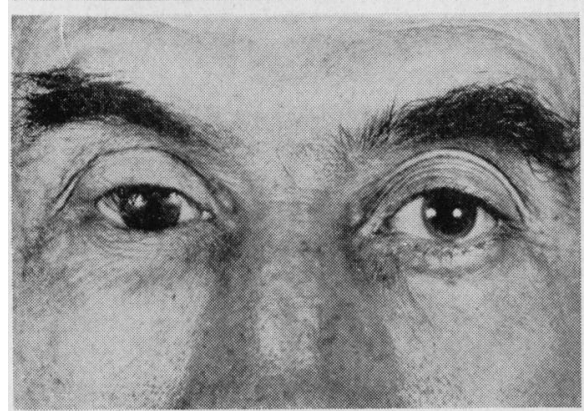

(B) Patient after treatment fitted with artificial eye.

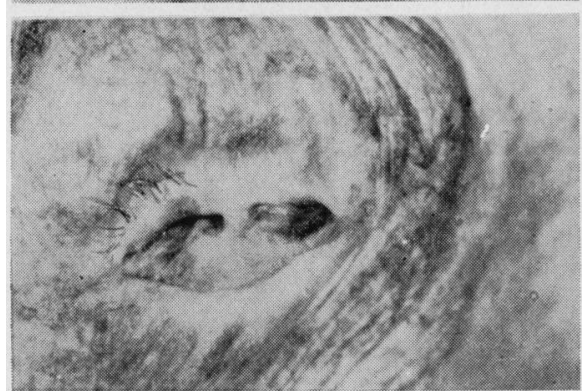

(C) Socket contraction and adhesion where glass shell was not worn during radiation treatment.

FIG. 22.-Post-operative irradiation of socket.

it can be prevented by making the patient wear a glass shell from the very beginning of treatment. It is surprising how well patients tolerate these shells in sockets which show severe reaction. Once the reaction has settled down and the socket has "stabilized" itself as far as contraction is concerned, the temporary glass shell can be replaced by the final artificial eye.

\section{Prevention of Ocular Complications}

Selection of Cases.-A good eye should never be sacrificed for the sake of technical expediency or fear of post-radiation complications, but in certain 15 
circumstances the eye should be removed as a preliminary to radiotherapy

(a) a blind eye which has already been damaged before treatment is begun,

(b) an eye which is proptosed and affected by a severe exposure keratitis, neuroparalytic keratitis, or a panophthalmitis.

In dealing with radio-sensitive tumours, even a severely damaged eye may be $\frac{\bar{C}}{0}$ saved, and this should always be borne in mind when a patient is reluctant to $\frac{\overline{\bar{c}}}{\overline{\frac{\pi}{5}}}$ agree to preliminary removal of the eye (Fig. 5).

\section{Technique}

(a) The greatest safeguard to the eye is to shield it against radiation either $\vec{\circ}$ directly or indirectly. The vulnerable part of the anterior segment of the eye $\vec{\omega}$ should be shielded in all cases, and the sound eye should be completely shielded $\stackrel{\omega}{\circ}$ as a routine however slight the apparent risk.

(b) Local contact methods of gamma radiation using radio-active sources near. the normal eye should be avoided, since they carry a high risk of ocular damage $\vec{\circ}$ and should with few exceptions be reserved for the treatment of the socket.

(c) Low dosage and the avoidance of brisk reactions will go far to eliminate 5 ocular complications in treating non-malignant tumours. Local tissue reactions $\overrightarrow{-}$ to radiation are inevitable in treating malignant tumours, but can in part be controlled by attention to the dosage and time factor.

Ophthalmological Control.-All patients receiving radiation in the region of $\overrightarrow{0}$ the eye should be seen by an ophthalmologist before, during, and after treatme Only by regular observation can incipient complications be detected and dealt: with promptly. The care of the eye during the stage of acute reaction is a majoro measure and requires both ophthalmological supervision and skilled nursing.

I am most indebted to the surgeons, both ophthalmologists and laryngologists, who have sento me cases and enabled the work to be carried out, particularly Mr. C. Dee Shapland, consultant $\odot$ ophthalmologist, and Mr. J. M. Mallett, ophthalmic registrar to the Eye Department of the Royal $\overrightarrow{\vec{\sigma}}$ Marsden Hospital, who have preserved many an eye from the depredations of radiotherapy. I3 am grateful to Dr. J. W. Whittick, Director of the Pathology Department of the Royal Marsden Hospital, and to Dr. Norman Ashton, Director of the Department of Pathology of the Institute of Ophthalmology, and also to Dr. J. Jackson Richmond, Director of the Radiotherapy Department of St. George's Hospital, for permission to include one successfully-treated lymphosarcema.

I also wish to thank several generations of assistants, in particular Dr. A. D. O'Connor, now of the Kent and Canterbury Hospital, Miss V. M. Dalley, my assistant for the past 6 years, and finally 3 . Miss L. J. Hunt, of the Department of Medical Photography, who provided the illustrations.

The Editor of the Proceedings of the Royal Society of Medicine has given me permission to draw upon material published in that Journal.

\section{REFERENCES}

DUKE-ELDER, S. (1952). “ "Text-book of Ophthalmology,” vol. 5. Kimpton, London. Euls, M. (1955). Brit. med. J., 1, 1251.

OFFRETT, G. (1951). "Les tumeurs primitives de l'orbite, leur traitement". Masson, Paris. ReEse, A. B. (1951). " "Tumours of the Eye". Cassell, London. 